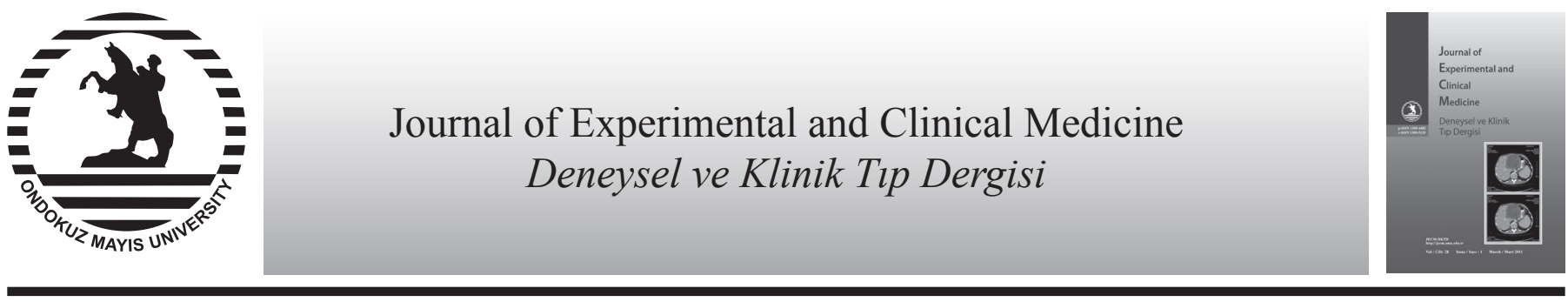

Clinical Research

\title{
The effect of hepatic hydatid cysts on the size of spleen
}

\author{
Kadir Diclea , Cafer Polat ${ }^{a}$, Bünyamin Şahin ${ }^{b}$, Bülent Güngör ${ }^{a}$, Ayfer Kamalı Polat ${ }^{a}$, Kenan Erzurumlu ${ }^{a}$ \\ ${ }^{a}$ Department of General Surgery, Medical Faculty, Ondokuz Mayis University, Samsun, Turkey \\ ${ }^{b}$ Department of Anatomy, Medical Faculty, Ondokuz Mayis University, Samsun, Turkey
}

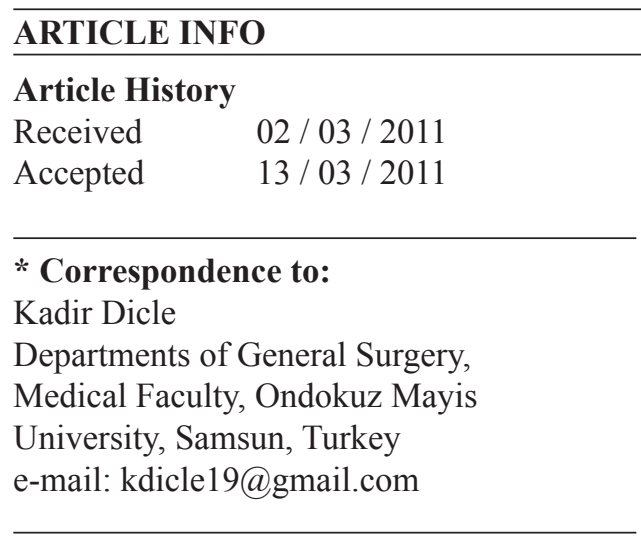

\section{Keywords:}

Hepatic hydatid cyst

Splenomegaly

Cavalieri principle

Stereology

Computed tomography

\begin{abstract}
No previous study has yet reported the effects of surgical treatment of hydatid cysts on spleen volume. Our aim was to use computed tomography images to evaluate the volume differences in the spleen in postoperative periods in patients who had undergone operations for hydatid cysts. The abdominal computed tomography scans of 20 patients who had undergone drainage of a hydatid cyst and omentopexy operation were examined retrospectively. The volume of the liver, the cyst, and the spleen were estimated using the Cavalieri principle of stereological methods. The volume fraction of the hydatid cyst to the liver was also estimated using the obtained volume data. The volumes of the spleen $( \pm \mathrm{SD})$ were $302.0 \pm 115.1 \mathrm{~cm}^{3}$ and $259.5 \pm 82.4 \mathrm{~cm}^{3}$ in the postoperative 1 st week and 6 months, respectively $(\mathrm{p}<0.05)$. The volumes of the liver $( \pm \mathrm{SD})$ were $2264.0 \pm 719.2$ $\mathrm{cm}^{3}$ and $1651.0 \pm 265.6 \mathrm{~cm}^{3}$ in the postoperative 1st week and 6 months, respectively $(p<0.05)$. The liver volume decreased significantly during the healing process $(p<0.05)$. The mean cyst volume was $417.4 \pm 649.9 \mathrm{~cm}^{3}$. The volume fraction of the cyst within the liver was $15.2 \pm 21.0 \%$. There were no correlations among liver, spleen, and cyst size of the patients $(\mathrm{p}>0.05)$. The volume of spleen decreased after hydatid cyst surgery. This may be due to the decreased portal venous pressure of the liver. Additionally, the loss of immune stimulation may have resulted in reduction in splenic volume. Our results also indicated that stereological methods can be used to monitor volume changes of the liver and spleen.

J. Exp. Clin. Med., 2011; 28:8-11
\end{abstract}

C 2011 OMU All rights reserved

\section{Introduction}

Hydatid disease is still an important health problem in developing countries, especially in sheep grazing areas. The echinococcal cysts can easily locate in all visceral organs, with cysts occurring most frequently in the liver. The pathophysiologic changes produced in the organism are still being researched. An enlarged intrahepatic cyst may cause compression on the surrounding structures or viscera, resulting in fibrosis and atrophy of the adjacent parenchyma of the liver (Dziri, 2001; Mc Manus et al., 2003; Dziri, 2004). Many surgeons have noted an enlargement of the spleen during hydatid cyst surgery. However, there is no reported splenomegaly associated with the hepatic hydatid cyst in the literature. Although it is not common, the pathogenesis of splenomegaly in hepatic hydatidosis is not yet clearly understood.

In the present stereological study, we estimated the volume differences in the spleen in postoperative periods using computed tomography (CT) images. In the light of the obtained data, we evaluated the effect of surgical treatment of hydatid cysts on the volume decrease in the spleen.

\section{Material and Methods}

The ethics board of the university approved the present retrospective study. The abdominal CT scans of 20 patients who had undergone drainage of the cyst and an omentopexy operation for the treatment of hydatid cyst were examined retrospectively. The volume of the liver, the cyst, and the spleen were estimated using the Cavalieri principle of stereological methods. The volume fraction of the hydatid cyst to the liver was also estimated using the obtained volume data.

The CT scans of patients were performed using a multislice CT machine (Toshiba TSX-101A, Aquilion 16 Slice, Tochigi, Japan). The scanning was performed with the following parameters: kVP: 120, mAs: 75, DFOV: $400 \mathrm{~mm}$, effective thickness: $5 \mathrm{~mm}$. The imaging data were stored as Dicom files.

The consecutive CT scans were converted into BMP formatted images using DicomWorks (Version 1.3.5) software. The images were transferred into ImageJ (Version 1.37) which is distributed freely by the National Institutes of Health, USA. First, the images were converted into stacks, then the program 
was matched using the reduction scale of the images. Since the consecutive images were more than required, a systematic random sampling procedure was used and every 1 out of 3 sections and 1 out of 2 sections were used for the liver and spleen volume estimations, respectively. Sectional cut surface areas of the liver and spleen were estimated using the planimetry method. For this purpose, the polygonal selection tool of the program was used and the contours of the liver and spleen were delineated. The software automatically calculated the number of pixels enclosed by the traced structure contours on each section and provided the cross-sectional area of the organ of interest on a slice-by-slice basis (Fig. 1). The sum of the areas multiplied by the section thickness provided the volume of structure, as shown in the formula (Sahin et al., 2007):

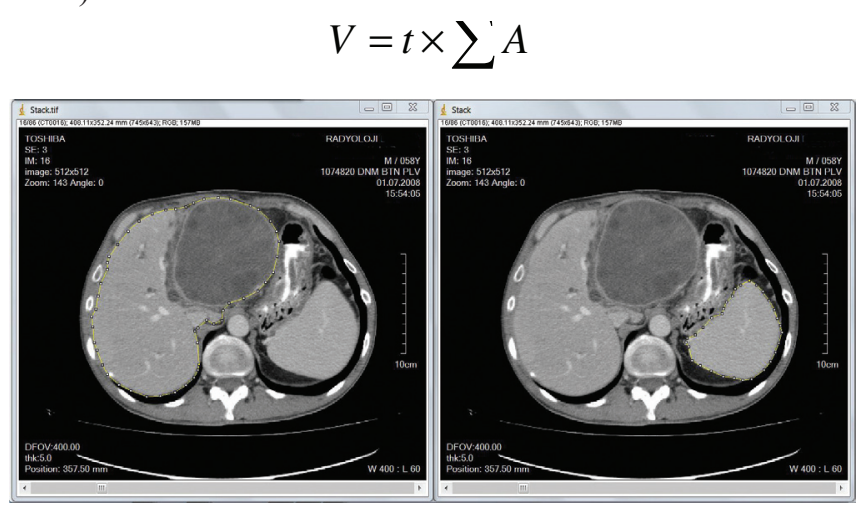

Fig. 1. Delineation of the boundaries of liver and spleen using ImageJ software to estimate the volume of the organs.

where $t$ is the section thickness of consecutive sections $(1 \mathrm{~cm}$ and $1.5 \mathrm{~cm}$ for the spleen and liver, respectively) and $\Sigma \mathrm{A}$ is the total sectional area of the consecutive sections.

The volume fraction of the cyst to the liver was estimated using the following formula (Basoglu et al., 2007):

$\mathrm{VV}($ cyst, liver $)=($ Volume of cyst $/$ volume of liver $) \times 100$

(Mc Manus et al., 2004)

The results of section cut surface areas were transferred into a Microsoft Excel worksheet and the volumes of liver, cyst, and spleen and the volume fraction of cysts to the liver were obtained automatically.

The same procedure was applied to CT scans that were obtained in the first week and sixth months after the operation. Obtained postoperative volume data were compared statistically using Wilcoxon Signed Ranks test. A p value equal or less than 0.05 was accepted as statistically significant.

\section{Results}

In the present study, we evaluated volume changes of the spleen in postoperative period. Our result showed that the volumes of spleen $( \pm \mathrm{SD})$ were $302.0 \pm 115.1 \mathrm{~cm}^{3}$ and 259.5 $\pm 82.4 \mathrm{~cm}^{3}$ in the postoperative $1 \mathrm{st}$ week and 6 th month, respectively. The volume differences of the spleen were statistically significant $(\mathrm{p}<0.05)$. The volumes of liver $( \pm \mathrm{SD})$ were $2264.0 \pm 719.2 \mathrm{~cm}^{3}$ and $1651.0 \pm 265.6 \mathrm{~cm}^{3}$ in the postoperative 1 st week and 6 th month, respectively. The liver volume decreased significantly during the healing process $(\mathrm{p}<0.05)$. The mean cyst volume was $417.4 \pm 649.9 \mathrm{~cm}^{3}$. The volume fraction of the cyst within the liver was $15.2 \pm 21.0 \%$. There were no correlations among liver, spleen and cyst size of the patients $(p>0.05)$. The details of these findings are summa- rized in Table 1.

Table 1. The quantitative findings of the liver, spleen and cyst. Volume, $\mathrm{cm}^{3}$; volume fraction, $\%$.

\begin{tabular}{|l|c|c|c|c|}
\hline & Mean & SD & Minimum & Maximum \\
\hline Volume of cyst & 417.4 & 647.9 & 16.6 & 2478.5 \\
\hline Volume fraction of cyst & 15.2 & 21.0 & 1.0 & 81.7 \\
\hline Postop spleen volume & $302.0^{*}$ & 115.1 & 170.7 & 590.2 \\
\hline $6^{\text {th }}$ month spleen volume & $259.5^{*}$ & 82.4 & 173.1 & 503.7 \\
\hline Postop liver volume & $2264.0 ¥$ & 719.2 & 1488.0 & 3997.8 \\
\hline $6^{\text {th }}$ month liver volume & $1651.0 ¥$ & 265.6 & 1200.0 & 2285.4 \\
\hline
\end{tabular}

\section{Discussion}

Hydatid disease is a parasitic infection. The cysts may reach to great sizes in the visceral organs and therefore have the potential to produce changes in adjacent tissues and organs. The splenic enlargement in hydatid disease of the liver was the focus of our study. In addition to portal hypertension, pathologies that affect the liver generally result in splenomegaly. However, evaluation of splenomegaly using palpation is difficult and may lead to inaccurate diagnosis (De Odorico et al., 1999). Accurate measurement of splenic volume is an important parameter in diagnosing splenomegaly and in improving therapeutic management of patients (Mazonakis et al., 2000).

Accurate information about the actual volume of liver and spleen cannot be obtained by routine physical examination. However, the coupling of physical examination with diagnostic imaging for clinical diagnosis remains insufficient. The liver volume has been shown to be useful in prognosis of patients with cirrhosis, but its measurement needs to be quantitative and reproducible, which can be obtained only by imaging techniques (Zoli et al., 1995).

A simple, inexpensive stereological approach was shown in the present study to be well suited to rapid and accurate volumetric calculations on the basis of standard sectional radiological techniques of liver that could be affected by tumors, cirrhosis, hepatitis, hepatomegaly, liver transplantation, hepatectomy and other surgical applications. It is also useful to examine structures that require assessment of changes in volume over time as an indicator of therapeutic effectiveness (Yamanaka et al., 1993; Schiano et al., 2000; Vogl et al., 2000).

The requirement for the application of the Cavalieri principle is an entire set of two-dimensional slices through the object. These need to be parallel, separated by a known distance, and begin randomly within the object, which are criteria that are met by standard sectional radiological imaging techniques (Cruz-Orive, 1997; Roberts et al., 2000; Sahin et al., 2003). The section thickness of the images is multiplied by the cut surface area of the sections and the total volume of the object of interest is estimated. The cut surface areas of the sections can be estimated using the planimetry technique that is used for estimating volume based on the Cavalieri principle (Mazonakis et al., 2004; Sahin and Ergur, 2006).

In the current study, manual planimetry was used to measure the volume of liver, cyst, and spleen. Taking into account the need for manual delineation of the contours of the struc- 
ture of interest on a section-by-section basis, it is true that the volumetric method is laborious. However, planimetric measurements are currently considered reference values in many volumetric studies (Bentley and Karwoski,1988; Dachman et al., 2001; Mazonakis et al., 2004), because they can provide accurate results whenever the user has the ability to precisely trace organ boundaries and knows the regional anatomy and morphology.

Splenomegaly is an expected finding in hydatid cyst disease of the spleen. In 2.5-3\% of the patients with hydatid disease, the enlargement of the spleen may be determined. In related series, this ratio goes up to the $7.69 \%$ (Ammann and Eckert, 1996; Hokelek et al., 2002; Kern, 2003; Eckert and Deplazes, 2004; Moro PL, Schantz, 2006; Junghanss et al., 2008; Thompson, 2008). Our findings revealed that the volume of the spleen decreased about $15 \%$ six months after the hydatid cyst operation.

When the extrasplenic involvement of the hydatid cyst occurs, we also could observe splenomegaly of different proportions. Immune parameters may be related with the formation of splenomegaly when hydatid cysts involve distant organs like lung, brain, and bones. Antibody levels (IgG, IgM, and $\mathrm{IgE}$ ) in humans are frequently elevated in ecchinococcosis infections. Circulating parasite antigens are collected by specific antibodies and in some seronegative patients, detection of both circulating antigens and immune complexes support this finding.

Although a direct immunological phenomenon has not been defined for the death of the cysts, this may probably occur due to the immune responses of the host. Transfer of sec- ondary cysts from mouse to mouse has been shown to cause death in more than $40 \%$ of the cysts. This finding supports the hypothesis that cysts may be killed by immune parameters (Ammann and Eckert, 1996). The immune response related to these antigenic properties of the hydatid cysts confirms the spleen as a major component of the reticuloendothelial system that has a tendency to increase in function and volume.

The increased portal vein pressure as a result of extended periportal inflammation and/or fibrosis related with huge or multiple cysts may cause congestive splenomegaly. Cysts located very close to the porta hepatis or very large cysts compressing the portal vein may be responsible for congestive splenomegaly. In both situations, the splenomegaly related to the slowly developing and compensated portal hypertension may not produce any clinical symptoms. As well, the symptoms may not be obvious because of the symptoms related to the effects of the cyst on the liver. Regarding this phenomenon, portal hypertension and congestive splenomegaly are expected decrease after the aspiration or resection of the liver cyst. In our study, this would explain the decreased volume of the spleen after surgical treatment of the liver cysts depending on these pathophysiologic events.

In conclusion, we found that the volume of the spleen decreased after surgery of hepatic hydatid cysts. This may be due to the decreased portal venous pressure of the liver. The loss of immune stimulation may then result in the reduction of splenic volume. Stereological methods can provide quantitative and reliable data for monitoring size changes such as these in the liver and spleen.

\section{REFERENCES}

Ammann, R.W., Eckert, J. 1996. Cestodes: Echinococcus. Gastro. Clin. North America. 25, 655-689.

Başoğlu, A., Büyükkarabacak, Y., Sahin, B., Kaplan, S. 2007. Volumetric evaluation of the lung expansion following resection: a stereological study. Eur. J. Cardiothorac. Surg. 31, 512-517.

Bentley, M.D., Karwoski, R.A. 1988. Estimation of tissue volume from serial tomographic sections. A statistical random marking method. Invest Radiol. 23, 742-747.

Cruz-Orive, L.M. 1997. Stereology of single objects. J. Microsc. 186, 93-107.

Dachman, A.H., MacEneaney, P.M., Adedipe, A., Carlin, M., Schumm, L.P. 2001. Tumor size on computed tomography scans: is one measure ment enough? Cancer. 91, 555-560.

De Odorico, I., Spaulding, K.A., Pretorius, D.H., Lev-Toaff, A.S., Bailey, T.B., Nelson, T.R. 1999. Normal splenic volumes estimated using three-dimensional ultrasonography. J. Ultrasound. Med. 18,231-236.

Dziri, C. 2001. Hydatid Disease: Continuous serious public health problem. World J. Surg. 25,1-3.

Dziri, C., Haouet, K., Fingerhut, A. 2004. Treatment of hydatid cyst of the liver: Where is the evidence? World J. Surg. 28,731-736.

Eckert, J., Deplazes, P. 2004. Biological, epidemiological, and clinical aspects of echinococcosis, a zoonosis of increasing concern. Clin. Microbiol. Rev. 17, 107-135.

Hokelek, M., Deger, B.A., Deger, E., Tutar, E., Sunbul, M. 2002. Ivermectin used in percutaneous drug injection method for the treatment of liver hydatid disease in sheep. Gastroenterology. 122, 957-962.

Junghanss, T., da Silva, A.M., Horton, J., Chiodini, P.L., Brunetti, E. 2008. Clinical management of cystic echinococcosis: state of the art, prob lems, and perspectives. Am. J. Trop Med. Hyg. 79, 301-311.

Kern, P. 2003. Echinococcus granulosus infection: clinical presentation, medical treatment and outcome. Langenbecks Arch. Surg. 388,413420 .

Mazonakis, M., Damilakis, J., Maris, T., Prassopoulos, P., Gourtsoyiannis, N. 2000. Estimation of spleen volume using MR imaging and a ran dom marking technique. Eur. Radiol. 10,1899-1903.

Mazonakis, M., Damilakis, J., Mantatzis, M., Prassopoulos, P., Maris, T., Varveris, H., Gourtsoyiannis, N. 2004. Stereology versus planimetry toestimate the volume of malignant liver lesions on MR imaging. Magn. Reson. Imaging. 22,1011-1016.

Mc Manus, D.P., Zhang, W., Li, J., Bartley, P.B. 2003. Echinococcus. Lancet. 362, 1295-1304.

Moro, P.L., Schantz, P.M. 2006. Echinococcosis: historical landmarks and progress in research and control. Ann Trop. Med. Parasitol. 100,703714.

Roberts, N., Puddephat, M.J., McNulty, V. 2000. The benefit of stereology for quantitative radiology. Br. J. Radiol. 73,679-97.

Sahin, B., Acer, N., Sonmez, O.F., Emirzeoglu, M., Basaloglu, H., Uzun, A., Bilgic, S. 2007. Comparison of four methods for the estimation of intracranial volume: a gold standard study. Clin. Anat. 20, 766-773.

Sahin, B., Emirzeoglu, M., Uzun, A., Incesu, L., Bek, Y., Bilgic, S., Kaplan, S. 2003. Unbiased Estimation of the liver volume by the Cavalieri Principle using magnetic resonance images. Eur. J. Radiol. 47,164-170. 
Sahin, B., Ergur, H. 2006. Assessment of the optimum section thickness for the estimation of liver volume using magnetic resonance images: A stereological gold standard study. Eur. J. Radiol. 57, 96-101.

Schiano, T.D., Bodian, C., Schwartz, M.E., Glajchen, N., Min, A.D. 2000. Accuracy and significance of computed tomographic scan assess ment of hepatic volume in patients undergoing liver transplantation. Transplantation. 69, 545-550.

Thompson, R.C. 2008. The taxonomy, phylogeny and transmission of echinococcus. Exp. Parasitol. 119, 439-446.

Vogl, T.J., Trapp, M., Schroeder, H., Mack, M., Schuster, A., Schmitt, J. Neuhaus, P., Felix, R. 2000. Transarterial chemoembolization for hepatocellular carcinoma: Volumetric and morphologic CT criteria for assessment of prognosis and therapeutic success-results from a liver transplantation center. Radiology. 214, 349-357.

Yamanaka, N., Okamoto, E., Kawamura, E., Kato, T., Oriyama, T., Fujimoto, J., Furukawa, K., Tanaka, T., Tomoda, F., Tanaka, W. 1993. Dy namics of normal and injured human liver regeneration after hepatectomy as assessed on the basis of computed tomography and liver function. Hepatology. 18, 79-85.

Zoli, M., Magalotti, D., Grimaldi, M., Gueli, C., Marchesini, G., Pisi, E. 1995. Physical examination of the liver: is it still worth it? Am. J. Gastroenterol. 90, 1428-1432. 